\title{
The Modified Response Evaluation Criteria in Solid Tumors (RECIST) Yield a More Accurate Prognoses Than the RECIST 1.1 in Hepatocellular Carcinoma Treated with Transarterial Radioembolization
}

\author{
Jae Seung Lee ${ }^{1,2}$, Hong Jun Choi ${ }^{1,2}$, Beom Kyung Kim ${ }^{1,2,3}$, Jun Yong Park ${ }^{1,2,3}$, Do Young Kim ${ }^{1,2,3}$, Sang Hoon Ahn ${ }^{1,2,3}$, \\ Kwang-Hyub Han ${ }^{1,2,3}$, Song-Ee Baek ${ }^{2,4}$, Yong Eun Chung ${ }^{3,4}$, Mi-Suk Park ${ }^{2,4}$, Myeong-Jin Kim ${ }^{2,4}$, Hyungjin Rhee ${ }^{2,4}$, \\ and Seung Up Kim ${ }^{1,2,3}$ \\ ${ }^{1}$ Department of Internal Medicine, Yonsei University College of Medicine, ${ }^{2}$ Yonsei Liver Center, Severance Hospital, ${ }^{3}$ Institute of \\ Gastroenterology and ${ }^{4}$ Department of Radiology, Yonsei University College of Medicine, Seoul, Korea
}

See editorial on page 671.

Background/Aims: The Response Evaluation Criteria in Solid Tumors (RECIST) 1.1 and modified RECIST (mRECIST) criteria have been used to assess treatment responses for hepatocellular carcinoma (HCC) patients. We investigated which criteria provides better survival predictions in HCC patients treated with transarterial radioembolization (TARE). Methods: In total, 102 patients with unresectable intrahepatic HCC, who were treated with TARE between 2012 and 2017, were reviewed retrospectively. The treatment response after TARE was evaluated at 1, 3, and 6 months by the mRECIST and RECIST 1.1. Responders were defined as patients with complete or partial responses by each criterion. Results: The median age of 83 men and 19 women was 64.3 years. The median alpha-fetoprotein and des-gamma-carboxy prothrombin levels were $37.1 \mathrm{ng} / \mathrm{mL}$ and 1,780.0 $\mathrm{mAU} / \mathrm{mL}$, respectively. The median maximal tumor size was $8.3 \mathrm{~cm}$, and multiple tumors were observed in 36 patients (35.3\%). During the follow-up period (median, 20.7 months), 21 patients (20.6\%) died, with a mean survival time of 55.5 months. The cumulative survival rate was $96.1 \%$ at 6 months and $89.3 \%$ at 12 months. Responders, defined by the mRECIST at 1, 3, and 6 months after TARE, showed better survival outcomes than nonresponders (hazard ratio $[\mathrm{HR}]=5.736, \mathrm{p}=0.008$ at 1 month; $\mathrm{HR}=3.145, \mathrm{p}=0.022$ at 3 months, and $\mathrm{HR}=2.887$, $p=0.061$ at 6 months). The survival rates of responders and nonresponders defined by the RECIST 1.1 were similar (all $p>0.05)$. Conclusions: Response evaluations that use the mRECIST provide more accurate prognoses than those that use the RECIST 1.1 in HCC patients treated with TARE. (Gut Liver 2020;14:765-774)

Key Words: Carcinoma, hepatocellular; Treatment outcome; Response Evaluation Criteria in Solid Tumors; Embolization, therapeutics; Radiotherapy

\section{INTRODUCTION}

Hepatocellular carcinoma (HCC) is one of the most common malignancies and a common cause of cancer-related death. ${ }^{1}$ In 2016, mortalities from HCC ranked second among cancer deaths in South Korea. ${ }^{2}$ Many patients are diagnosed at advanced stages and are not candidates for curative treatment because of old age, co-morbid diseases, portal hypertension, liver dysfunction with cirrhosis, multiple tumors, portal vein invasion, or inability to secure a sufficient resection margin.,

Recently, the usefulness of transarterial radioembolization (TARE) for intrahepatic HCC has been reported. ${ }^{5-8}$ TARE is a liver-directed localized internal radiation therapy that uses an microembolic procedure for liver malignancies. It involves intraarterial injection of radioactive substances, such as yttrium-90 $\left({ }^{90} \mathrm{Y}\right)$-loaded microspheres, to minimalize hepatic arterial flow alterations. ${ }^{5,9}$ TARE has showed a good safety profile, significantly longer time to progression (TTP), better tumor control,

Correspondence to: Seung Up Kim ${ }^{a}$ and Hyungjin Rhee ${ }^{b}$

${ }^{a}$ Department of Internal Medicine, Yonsei University College of Medicine, 50 Yonsei-ro, Seodaemun-gu, Seoul 03722, Korea

Tel: +82-2-2228-1930, Fax: +82-2-393-6884, E-mail: ksukorea@yuhs.ac

${ }^{b}$ Department of Radiology, Yonsei University College of Medicine, 50 Yonsei-ro, Seodaemun-gu, Seoul 03722, Korea

Tel: +82-2-2228-7408, Fax: +82-2-2227-8337, E-mail: hjinrhee@yuhs.ac

Received on June 5, 2019. Revised on November 21, 2019. Accepted on November 29, 2019. Published online February 14, 2020.

pISSN 1976-2283 eISSN 2005-1212 https://doi.org/10.5009/gnl19197

Jae Seung Lee and Hong Jun Choi contributed equally to this work as first authors.

@) This is an Open Access article distributed under the terms of the Creative Commons Attribution Non-Commercial License (http://creativecommons.org/licenses/by-nc/4.0) which permits unrestricted non-commercial use, distribution, and reproduction in any medium, provided the original work is properly cited. 
and higher quality of life. Thus, TARE can be an alternative treatment for patients with intermediate and advanced stages, who have a risk of procedure-related liver failure and complications after transarterial chemoembolization (TACE), ${ }^{5-8,10,11}$ and can be used to achieve a complete cure or as a bridging therapy for other curative options that reduce recurrence risk. ${ }^{12,13}$

Tumor responses have been commonly measured according to the World Health Organization criteria and the Response Evaluation Criteria in Solid Tumors version 1.1 (RECIST 1.1) criteria, which are based on tumor size and number. ${ }^{14,15}$ However, modified RECIST (mRECIST) criteria has recently been adopted for HCC, and this criteria considers the concepts of tumor viability based on arterial enhancement and single linear summation, which ultimately simplify European Association for the Study of the Liver (EASL) criteria. ${ }^{15-19}$ Nowadays, mRECIST is widely used as a response evaluation method for several HCC therapies and provides good prognostic value for predicting overall survival (OS). ${ }^{20-23}$ However, the best response evaluation method for predicting treatment outcomes of HCC after TARE treatment remains controversial. ${ }^{6,24-27}$

In this study, we investigated the long-term outcomes according to treatment response using RECIST 1.1 and mRECIST criteria, and compared the prognostic accuracy of both RECIST criteria for predicting OS of HCC patients treated with TARE using ${ }^{90} \mathrm{Y}$.

\section{MATERIALS AND METHODS}

\section{Patient eligibility}

Between 2011 and 2017, HCC patients treated with TARE were recruited using a retrospective review of the patient database at Yonsei Liver Center, Severance Hospital, Yonsei University College of Medicine.

The exclusion criteria were (1) age under 19 years; (2) Eastern Cooperative Oncology Group Performance Status >2; (3) extrahepatic HCC; (4) presence of ascites; (5) significant extrahepatic disease representing an imminent life-threatening outcome; (6) uncontrolled medical co-morbidities; (7) mortality of unknown cause that was not due to illness; (8) mortality that was absolutely due to procedure-related complications; (9) follow-up loss or death within 3 months after TARE; (10) other treatment modalities such as TACE, intra-arterial chemotherapy, and surgical resection within 6 months after TARE treatment, or large lipiodol uptake due to previous TACE that confounded response evaluation (Fig. 1).

The study protocol was in accordance with the 1975 Declaration of Helsinki guidelines. The need for written informed consent was waived because of the retrospective nature. The study procedure was approved by the institutional review board of Yonsei University Health System (IRB number: 4-2019-0035).
146 Patients with HCC who received TARE between 2011 and 2017
19 Patients were excluded due to

- Age under 19

- ECOG >2

- Extrahepatic HCC

- Presence of ascites

- Significant extrahepatic diseases

- Uncontrolled medical co-morbidities

127 Patients with intrahepatic HCC
25 Patients were excluded due to

- Mortality of unknown causes

- Mortality absolutely due to procedure-related complications

- Follow-up loss or expired within 3 month

- Other intra-arterial treatment or resection after TARE

- Too large lipiodol uptake due to previous TACE treatment that confounded the response evaluation
Fig. 1. Flowchart of the study population selection.

HCC, hepatocellular carcinoma; TARE, transarterial radioembolization; ECOG, Eastern Cooperative Oncology Group; TACE, transarterial chemoembolization. 


\section{HCC diagnosis}

HCC was diagnosed histologically or clinically, according to the guidelines proposed by the Korea Liver Cancer Study Group. $^{28,29}$ A positive finding for typical HCC on dynamic computed tomography or magnetic resonance imaging was described as an increased arterial enhancement followed by a decreased enhancement, compared to the liver (washout), in the

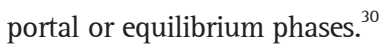

\section{Treatment modality}

Pretreatment angioscintigraphy with technetium-99 macroaggregated albumin scanning was performed in all patients, and the lung shunt fraction was assessed to avoid the extrahepatic spread of microspheres labeled with ${ }^{90} \mathrm{Y}$. Two to 3 weeks after pretreatment, TARE was performed with resin (SIR-Spheres ${ }^{\circledR}$; Sirtex Medical, Sydney, Australia) or glass particles (TheraSphere $^{\circledR}$; Biocompatibles UK Ltd., Surrey, UK) loaded with ${ }^{90}$ Y. The dose was determined from the planning angiogram and prepared in the nuclear medicine department, according to the preparation guide provide by the manufacturer. The targetabsorbed radiation doses to the normal liver and lungs should not exceed 70 Gy and 25 Gy, respectively. TARE was conducted according to previous guidelines. ${ }^{31}$

\section{Assessment of the treatment responses using RECIST 1.1 and MRECIST}

The treatment responses were routinely assessed at 1, 3, and 5 to 6 months after TACE sessions, using liver dynamic CT or MRI with a physical examination and blood tests. To minimize the possibility of false categorizations, two independent radiologists analyzed the images. In cases of disagreement, consensus of more than two radiologists reached.

The mRECIST and RECIST 1.1 guideline was applied for response evaluation. ${ }^{16,32}$ RECIST 1.1 defines target lesions as lesions which can be measured in at least one-dimension as above $1 \mathrm{~cm}$ with a spiral CT scan at baseline and be suitable for repeat measurement, and target lesions for mRECIST should show arterial enhancement additionally. Therefore, the target lesion was determined based on lesion size, as a largest single lesion. ${ }^{21}$ An infiltrative lesion that could be compared by measurements before and after treatment was also regarded as a target lesion in this study. For a target lesion, the tumor response was defined by each criteria as a complete response (CR), partial response (PR), stable disease (SD) and progressive disease (PD). Like in literature, assessment of nontarget lesions included portal vein thrombosis, which was suspected malignant due to the closed location to the tumor in either criteria, or viability with arterial enhancement in mRECIST. Finally, overall response was determined by combining the evaluation of target and nontarget lesions according to each criteria (Supplementary Table 1).

\section{Definitions}

In addition to $\mathrm{CR}, \mathrm{PR}, \mathrm{SD}$, and PR, "best response" was defined as the most favorable response during 6 months after TARE treatment. "Responder" was defined as the sum of patients who showed either CR or PR, according to RECIST 1.1 and mRECIST, which is similar to the definition of objective response that is used in the literature. "Nonresponder" was defined as the sum of those who showed either SD or PD.

Table 1. Baseline Characteristics

\begin{tabular}{|c|c|}
\hline Variable & Value $(n=102)$ \\
\hline \multicolumn{2}{|l|}{ Demographic variable } \\
\hline Age, yr & $64.3(53.6-72.8)$ \\
\hline Male sex & $83(81.4)$ \\
\hline Body mass index, $\mathrm{kg} / \mathrm{m}^{2}$ & $23.9(21.3-25.8)$ \\
\hline Diabetes mellitus & $44(43.1)$ \\
\hline Hypertension & $59(57.8)$ \\
\hline Viral etiology & $71(69.6)$ \\
\hline Liver cirrhosis & $28(27.5)$ \\
\hline \multicolumn{2}{|l|}{ Laboratory variables } \\
\hline Platelet count, $\times 10^{9} / \mathrm{L}$ & $198.5(121.3-261.5)$ \\
\hline Total bilirubin, mg/dL & $0.6(0.5-0.9)$ \\
\hline Serum albumin, g/dL & $3.9(3.5-4.1)$ \\
\hline Aspartate aminotransferase, IU/L & $40.0(28.8-64.3)$ \\
\hline Alanine aminotransferase, IU/L & $28.5(18.0-45.0)$ \\
\hline Alkaline phosphatase, IU/L & $95.5(76.0-138.5)$ \\
\hline Prothrombin time, INR & $1.02(0.96-1.10)$ \\
\hline AFP, ng/mL & $37.1(5.0-2,590.1)$ \\
\hline$>200$ & $39(38.2)$ \\
\hline$>400$ & $36(35.3)$ \\
\hline $\mathrm{DCP}, \mathrm{mAU} / \mathrm{mL}$ & $1,780.0(135.5-8,119.5)$ \\
\hline$>13,000$ & 19 (18.6) \\
\hline \multicolumn{2}{|l|}{ Tumor characteristics } \\
\hline Nodular/infiltrative & $82(80.4) / 20(19.6)$ \\
\hline Maximum tumor size, $\mathrm{cm}$ & $8.3(6.0-10.5)$ \\
\hline Multiple tumors & $36(35.3)$ \\
\hline Above 3 tumors & $26(25.5)$ \\
\hline Tumor burden $>50 \%$ & $10(9.8)$ \\
\hline Bilobar distribution & $28(27.5)$ \\
\hline Portal vein thrombosis & 19 (18.6) \\
\hline Segmentary order branch & $5(4.9)$ \\
\hline Second order branch & $6(5.9)$ \\
\hline First order branch & $8(7.8)$ \\
\hline Hepatic vein invasion & $5(4.9)$ \\
\hline
\end{tabular}

Data are presented as median (interquartile range) or number (\%). INR, international normalized ratio; AFP, alpha-fetoprotein; DCP, des-gamma-carboxyprothrombin. 


\section{Statistical analysis}

The Student t-tests and Mann-Whitney tests were used to compare quantitative variables. The chi-square tests and Fisher exact tests were used to compare qualitative variables. OS and progression-free survival (PFS) were calculated using the Kaplan-Meier analysis, and Cox proportional hazards model and a log-rank test that used Kaplan-Meier analysis were used to compare the hazard ratios between the results of the response evaluations at 1, 3, and 6 months after the initial TARE treatment. The criteria with a higher chi-square value by the likelihood ratio and linear trend tests were considered the better model for homogeneity and discriminatory ability. Furthermore, lower values for Akaike information criteria were considered better for discriminatory abilities. A p $<0.05$ was considered statistically significant, with a confidence interval of 95\%. Statistical analyses were conducted using the IBM SPSS statistics software version 23.0 (IBM Corp., Armonk, NY, USA).

\section{RESULTS}

\section{Patient characteristics}

The workflow the study population selection is depicted in Fig. 1. In total, 146 patients were considered eligible for this study. After excluding 44 patients who met our exclusion criteria, 102 patients who were treated with TARE for HCC were included in the analysis.

The baseline characteristics of the study population (83 men [81.4\%] and 19 women [18.6\%]) at the time of TARE treatment are shown in Table 1. The median patient age was 64.3 years. Seventy-one patients (69.6\%) had viral hepatitis and 28 patients (27.5\%) had liver cirrhosis. All patients had an Eastern Cooperative Oncology Group Performance Status score of 0 or 1.

The median alpha-fetoprotein level was $37.1 \mathrm{ng} / \mathrm{mL}$. In total, $39(38.2 \%)$ and 36 (35.3\%) patients had alpha-fetoprotein levels $>200 \mathrm{ng} / \mathrm{mL}$ and $>400 \mathrm{ng} / \mathrm{mL}$, respectively. The medial desgamma-carboxy prothrombin level was 1,708.0 mAU/mL. Nineteen patients (18.6\%) had des-gamma-carboxy prothrombin levels $>13,000 \mathrm{ng} / \mathrm{mL}$.

The median diameter of the largest measurable lesion was 8.3 $\mathrm{cm}$. Thirty-six patients (35.3\%) had multiple tumors, of whom $26(25.5 \%)$ had more than three tumors. Tumor invasion to peripheral branches of the portal vein was identified in 19 patients $(18.6 \%)$. Hepatic vein invasion was identified in five patients (4.9\%).

\section{Survival outcomes after TARE}

During the follow-up period (mean, 27.1 months; median, 20.7 months), 21 patients (20.6\%) died, with a mean survival of 55.5 months (Supplementary Fig. 1). The cumulative survival rate was $96.1 \%$ at 6 months and $89.3 \%$ at 12 months.

\section{Survival outcomes according to treatment response by RECIST 1.1 after TARE}

The survival outcomes according to treatment response by RECIST 1.1 after TARE treatment are summarized in Supplementary Table 2, and the corresponding Kaplan-Meier curves are described in Supplementary Fig. 2. The mean OS of two

Table 2. Overall Survival Analysis of Responders and Nonresponders According to the RECIST 1.1 and mRECIST

\begin{tabular}{|c|c|c|c|c|c|c|c|}
\hline Response criteria & Time point & Response & No. of patient & $\begin{array}{l}\text { Mean OS } \\
(95 \% \text { CI) }\end{array}$ & $\begin{array}{c}\text { p-value } \\
\text { (log-rank) }\end{array}$ & $\begin{array}{c}\text { Univariate HR } \\
\text { (95\% CI) }\end{array}$ & p-value \\
\hline \multirow[t]{8}{*}{ RECIST 1.1} & At 1 month & Responders & 2 & Not reached & 0.427 & - & \\
\hline & & Nonresponders & 99 & $55.0(48.6-61.3)$ & & 21.072 (too wide) & 0.598 \\
\hline & At 3 months & Responders & 13 & $64.9(55.2-74.6)$ & 0.130 & - & \\
\hline & & Nonresponders & 81 & $52.6(45.4-59.8)$ & & $4.208(0.557-31.779)$ & 0.164 \\
\hline & At 6 months & Responders & 20 & $60.0(50.1-70.0)$ & 0.291 & - & \\
\hline & & Nonresponders & 51 & $54.6(45.4-63.8)$ & & $2.013(0.536-7.561)$ & 0.300 \\
\hline & Best response & Responders & 23 & $60.9(51.6-70.2)$ & 0.115 & - & \\
\hline & & Nonresponders & 79 & $53.4(45.9-61.0)$ & & $2.596(0.759-8.881)$ & 0.128 \\
\hline \multirow[t]{8}{*}{ mRECIST } & At 1 month & Responders & 31 & $61.4(55.9-66.9)$ & 0.008 & - & \\
\hline & & Nonresponders & 70 & $49.1(40.8-57.5)$ & & $5.736(0.133-24.676)$ & 0.019 \\
\hline & At 3 months & Responders & 47 & $60.4(52.5-68.2)$ & 0.022 & - & \\
\hline & & Nonresponders & 47 & $48.4(38.8-58.0)$ & & $3.145(1.118-8.845)$ & 0.030 \\
\hline & At 6 months & Responders & 42 & $60.2(52.2-68.2)$ & 0.061 & - & \\
\hline & & Nonresponders & 29 & $47.3(35.0-59.6)$ & & 2.887 (0.907-9.187) & 0.073 \\
\hline & Best response & Responders & 58 & $61.3(54.7-67.9)$ & 0.001 & - & \\
\hline & & Nonresponders & 44 & $44.7(34.0-55.5)$ & & $4.594(1.775-11.893)$ & 0.002 \\
\hline
\end{tabular}

RECIST, Response Evaluation Criteria in Solid Tumors; mRECIST, modified RECIST; OS, overall survival; CI, confidence interval; HR, hazard ratio. 
patients (2.0\%) who showed PR at 1 month was not calculated, whereas those of SD $(n=96,94.1 \%)$ and PD patients $(n=3,2.9 \%)$ were 56.6 and 9.1 months, respectively. At 3 months, $P R(n=13$, 12.7\%), SD ( $n=69,67.6 \%)$, and PD patients ( $n=12,11.8 \%)$ showed mean OS values of 64.9, 56.2, and 26.1 months, respectively. At 6 months, PR ( $n=20,19.6 \%)$, SD $(n=39,38.2 \%)$, and PD patients ( $\mathrm{n}=12,11.8 \%$ ) showed mean OS values of 60.0, 62.7 and 17.0 months, respectively. Regarding the best response, PR $(\mathrm{n}=23,22.5 \%)$, SD $(\mathrm{n}=75,73.5 \%)$, and PD patients $(\mathrm{n}=4,3.9 \%)$ showed mean OS values of 60.9, 55.4, and 6.1 months, respectively. No significant differences were noted in the mean OS values between PR and SD at 1, 3, and 6 months, and in best responses (all p>0.05), whereas PD patients consistently showed significantly lower mean OS values than the other patients (all $\mathrm{p}<0.05)$. Twenty-four patients $(23.5 \%)$ experienced PD by RECIST 1.1 during 6 months, and the cause of PD was intrahepatic and extrahepatic progression $(\mathrm{n}=24,100 \%)$ (Supplementary Table 3).

\section{Survival outcomes according to treatment response by mRECIST after TARE}

Survival outcomes according to treatment response by mRE-

A
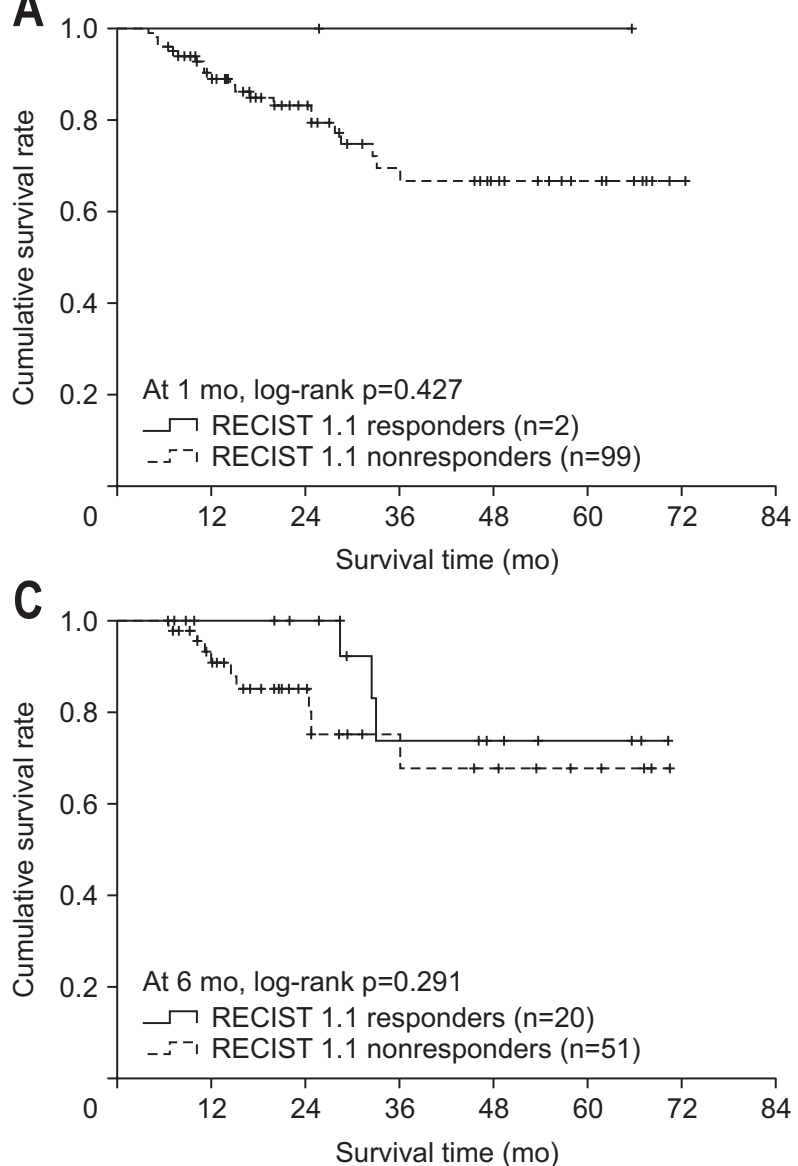

CIST 1.1 are summarized in Supplementary Table 2, and the corresponding Kaplan-Meier curves are described in Supplementary Fig. 3. The mean OS of three patients (2.9\%) who showed CR at 1 month after TARE was not calculated, whereas those of PR ( $n=28,27.5 \%)$, SD ( $n=67,65.7 \%)$, and PD patients $(\mathrm{n}=3,2.9 \%)$ were $60.9,51.3$, and 9.1 months, respectively. At 3 months, CR ( $n=12,11.8 \%)$, PR ( $n=35,34.3 \%)$, SD ( $n=33,32.4 \%)$, and PD patients $(n=14,13.7 \%)$ showed mean OS values of 56.3, 57.8, 55.7, and 25.0 months, respectively. CR patients at 3 months showed significantly higher OS than those with SD $(p=0.038)$; however, there was no significant difference in the mean OS between CR and PR $(\mathrm{p}=0.535)$ or between PR and SD $(\mathrm{p}=0.531)$. At 6 months, CR ( $\mathrm{n}=10,9.8 \%), P R \quad(n=32,31.4 \%), S D$ $(\mathrm{n}=12,11.8 \%)$, and PD patients $(\mathrm{n}=17,16.7 \%)$ showed mean OS values of 56.3, 58.3, 59.4, and 37.1 months, respectively, and there were no significant differences in $\mathrm{CR}, \mathrm{PR}$, and SD patients (CR vs PR, $\mathrm{p}=0.606$; CR vs PR, $\mathrm{p}=0.894$; PR vs SD, $\mathrm{p}=0.613$ ). Regarding the best response, CR $(n=14,13.7 \%)$, PR ( $n=44.4$, 43.1\%), SD ( $n=41,40.2 \%)$, and PD patients $(n=3,2.9 \%)$ showed mean OS values of 57.5, 59.2, 48.0, and 9.1 months, respectively. There were no significant differences in the mean OS values between CR and PR patients; however, CR and PR patients
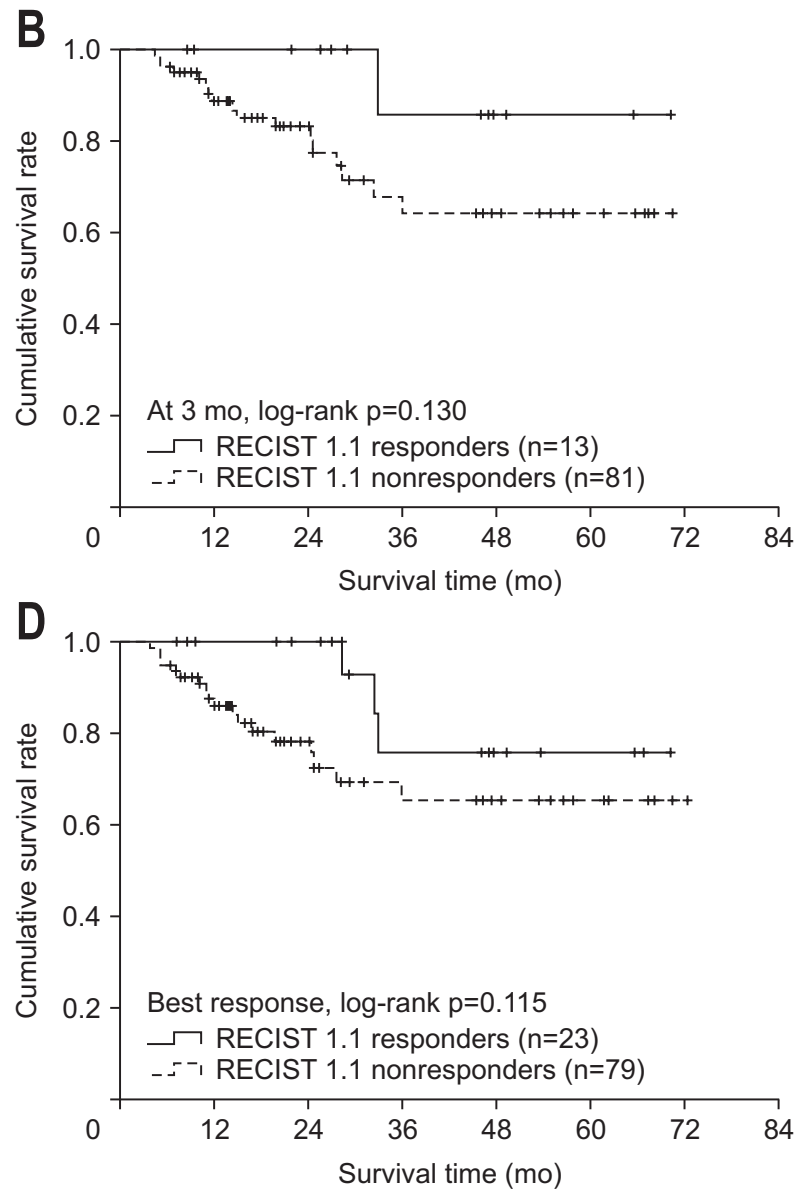

Fig. 2. Overall survival analysis of Response Evaluation Criteria in Solid Tumors (RECIST) 1.1 responders and nonresponders using Kaplan-Meier analysis at 1 month (A), 3 months (B), and 6 months (C) and as the best response (D). 

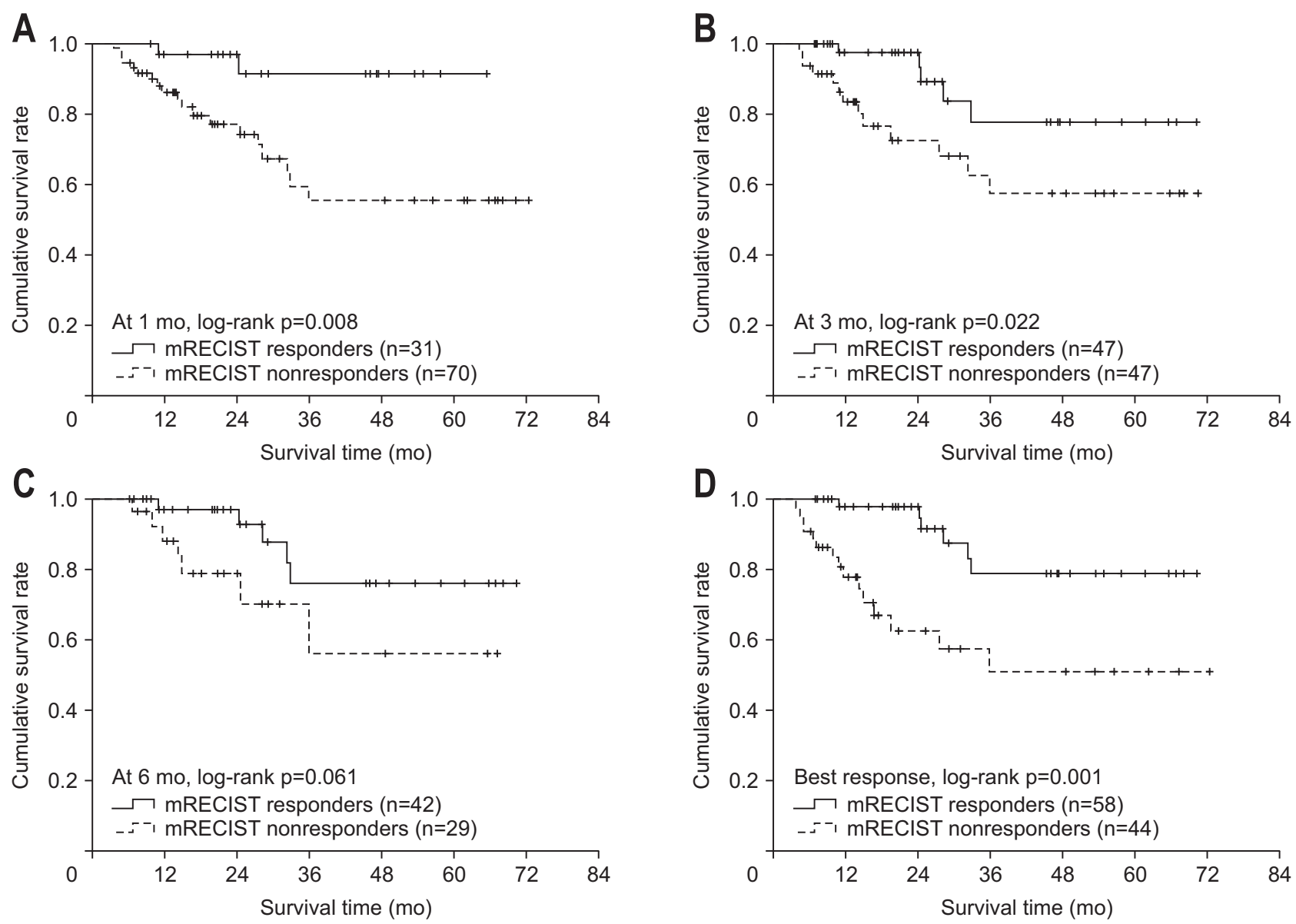

Fig. 3. Overall survival analysis of modified Response Evaluation Criteria in Solid Tumors (mRECIST) responders and nonresponders using KaplanMeier analysis at 1 month (A), 3 months (B), and 6 months (C) and as the best response (D).

Table 3. Prognostic Accuracy of the mRECIST and RECIST 1.1 to Predict Mortality after Transarterial Radioembolization

\begin{tabular}{|c|c|c|c|c|c|c|}
\hline \multirow{2}{*}{ Time point } & \multicolumn{2}{|c|}{ Likelihood ratio $\left(\chi^{2}\right)$} & \multicolumn{2}{|c|}{ Linear trend $\left(\chi^{2}\right)$} & \multicolumn{2}{|c|}{ AIC } \\
\hline & RECIST 1.1 & mRECIST & RECIST 1.1 & mRECIST & RECIST 1.1 & mRECIST \\
\hline At 1 months & $0.943(0.332)$ & $6.574(0.010)$ & $0.530(0.466)$ & $5.530(0.019)$ & 109.916 & 104.707 \\
\hline At 3 months & $1.529(0.216)$ & $4.527(0.033)$ & $1.265(0.261)$ & $4.351(0.037)$ & 96.117 & 92.901 \\
\hline At 6 months & $0.073(0.787)$ & $1.797(0.180)$ & $0.071(0.790)$ & $1.802(0.179)$ & 68.051 & 66.270 \\
\hline At best response & $1.119(0.290)$ & $8.679(0.003)$ & $1.024(0.312)$ & $8.544(0.003)$ & 109.705 & 101.728 \\
\hline
\end{tabular}

The model with a higher chi-square value by the likelihood ratio test and the linear trend test was considered the better model for homogeneity and discriminatory ability. Furthermore, lower values for Akaike information criteria (AIC) were considered better for discriminatory ability. Likelihood ratio and linear trend were shown as chi-square (p-value).

RECIST, Response Evaluation Criteria in Solid Tumors; mRECIST, modified RECIST.

showed significantly higher OS rates than SD patients $(\mathrm{p}=0.048$ and $\mathrm{p}=0.020$, respectively). PD patients consistently showed significantly lower mean OS values than the other patients (all $\mathrm{p}<0.05)$. Thirty patients $(29.4 \%)$ experienced PD by mRECIST during 6 months. The most common cause of PD by mRECIST at any time points $(n=30)$ was intrahepatic and extrahepatic progression ( $n=28,93.3 \%$ ), and local control failure was infrequent in patients with PD ( $\mathrm{n}=2,6.6 \%)$ (Supplementary Table 3).

\section{Better survival outcomes in responders}

Survival outcomes of responders and nonresponders after TARE, according to RECIST 1.1 and mRECIST at each time point, are summarized in Table 2.

The mean OS values of the responders versus nonresponders according to RECIST 1.1 were statistically similar at 1 month (not reached vs 55.0 months, $\mathrm{p}=0.427$ ), 3 months (64.9 months vs 52.6 months, $\mathrm{p}=0.130$ ), and 6 months ( 60.0 months vs 54.6 
months, $p=0.291$ ) after TARE. There was no significant difference in mean OS between responders and nonresponders according to the best response by RECIST 1.1. (60.9 months vs 53.4 months, $\mathrm{p}=0.115$ ). Accordingly, no significantly different relative risk of deaths between responders and nonresponders was observed (all p>0.05) according to RECIST 1.1 (Table 2, Fig. 2).

The mean OS of the responders was significantly higher or tended to be higher than that of nonresponders according to mRECIST (61.4 months vs 49.1 months at 1 month, $\mathrm{p}=0.008$; 60.4 months vs 48.4 months at 3 months, $\mathrm{p}=0.022 ; 60.2$ months vs 47.3 months at 6 months, $\mathrm{p}=0.061$; and 61.3 months vs 44.7 months regarding the best response, $p=0.001)$. The relative risk of death in nonresponders was significantly higher or tended to be higher than that of responders (unadjusted hazard ratio, 2.887 to 5.736: $p=0.019$ at 1 month, $p=0.030$ at 3 months, $p=0.073$ at 6 months, and $\mathrm{p}=0.002$ with the best response) (Table 2, Fig. 2).

Regarding the best response, nonresponders according to mRECIST showed its association with OS in multivariate analysis (adjusted hazard ratio, 3.432; $\mathrm{p}=0.015$ ), with other variables such as tumor distribution, first branch portal vein thrombosis and hepatic vein invasion (Supplementary Tables 4, 5).

\section{Prognostic performance of RECIST 1.1 and mRECIST re- sponders}

The prognostic performance of RECIST 1.1 and mRECIST to predict mortality was compared (Table 3). The likelihood ratios of RECIST 1.1 were not significant (0.943 at 1 month, 1.529 at 3 months, 0.073 at 6 months, and 1.119 regarding the best response; all $\mathrm{p}>0.05)$. In contrast, the likelihood ratios of mRECIST at 1 and 3 months were $6.574(\mathrm{p}=0.010)$ and $4.527(\mathrm{p}=0.033)$, respectively, with a best response of 1.797 ( $\mathrm{p}=0.180)$. Regarding the best response according to mRECIST, the likelihood ratio was 8.679 ( $p=0.003)$. Similarly, the linear trend of RECIST 1.1 was not significant (0.530 at 1 month, 1.265 at 3 months, 0.071 at 6 months, and 1.024 regarding the best response; all $\mathrm{p}>0.05$ ). In contrast, the corresponding linear trend of mRECIST 5.53 at 1 month ( $p=0.019), 4.527$ at 6 months $(p=0.037)$, and 1.802 for best response, with borderline statistical significance ( $p=0.179$ ). Regarding the best response according to mRECIST, the likelihood ratio and the linear trend were 8.679 and 8.544 (all $\mathrm{p}=0.003$ ), respectively. The likelihood ratio and the linear trend tended to be higher in mRECIST than RECIST 1.1 at each time point. The Akaike information criteria values of mRECIST were lower than those of RECIST 1.1 (104.707 vs 109.916 at 1 month; 92.901 vs 96.117 at 3 months; 66.270 vs 68.051 at 6 months; 101.728 vs 109.705 regarding the best response).

\section{PFS after TARE}

PFS was longer in responders according to both criteria ( $\mathrm{p}=0.043$ in RECIST 1.1 and $\mathrm{p}=0.005$ in mRECIST) (Supplementary Figs 4,5$)$. Regarding the best response before progression, nonresponders according to mRECIST showed its association with PFS in multivariate analysis (adjusted hazard ratio, 2.151; $\mathrm{p}=0.014$ ), while those according to RECIST 1.1 did not (Supplementary Tables 6-9).

\section{DISCUSSION}

TARE reduces tumor size and arterial enhancement on dynamic imaging, which indicate tumor shrinkage and necrosis, in a manner similar to TACE. ${ }^{27}$ Furthermore, TARE has been shown to be a safe and efficacious treatment strategy compared with conventional TACE, with a decreased risk of adverse events, an enhanced response rate, and a prolonged TTP., ${ }^{6,11}$ To decide whether we should further inspect patients for a delayed response to TARE or switch to another treatment modality is of paramount importance. To prevent disease progression, we must be able to discriminate the confounding responses that are due to the specific treatment mechanism of TARE (e.g., posttreatment inflammation, transient increase in lesion size associated with successful necrosis, peritumoral changes, and hemorrhage in the necrotic tumor) from true responses that indicate tumor reduction. ${ }^{26,27}$

In the present study, at early time points (1 or 3 months), the proportions of responders using RECIST 1.1 were significantly lower than using mRECIST. This indicates a clear methodological difference between the size-based RECIST 1.1 and enhancement-based mRECIST criteria. PD, as assessed by RECIST 1.1 and mRECIST, was significantly associated with poor survival at all-time points after TARE (all p<0.05). Furthermore, there were no significant difference in OS between responders and nonresponders by RECIST 1.1, and responders, as defined by mRECIST at 1 and 3 months, showed significantly better survival than nonresponders (all $\mathrm{p}<0.05$ ). In addition, prognosis stratification by mRECIST showed significantly higher chi-square values and lower Akaike information criteria values than by RECIST 1.1 at all-time points, which suggests the superior prognostic accuracy of mRECIST over RECIST 1.1.

Our study has several clinical implications. First, our results directly show that mRECIST criteria are superior to RECIST 1.1 for evaluating patient prognosis after TARE, despite the usefulness of enhancement criteria such as EASL and mRECIST after TARE has been controversial. Salem et al. ${ }^{6}$ evaluated long-term outcomes after TARE for HCC and noted that objective responders by EASL criteria showed better median OS predictions. Furthermore, Weng et al. ${ }^{24}$ noted that based on the responders at 3 months after TARE, by Choi criteria, the tumor density was a superior to predictor of TTP and OS. In this study, the responders at 3 months by mRECIST criteria also showed significance for TTP and OS ( $\mathrm{p}=0.004$ and $\mathrm{p}=0.077)$. Recently, Riaz et $a .^{25}$ performed 3-, 6-, and 12-month landmark analyses of 202 patients with solitary HCC and showed that the objective responders by EASL associated with longer survivals compared to size- 
based criteria. Our results also suggest that enhancement-based response evaluation criteria, such as mRECIST and EASL, might provide more detailed and accurate prognoses after TARE for HCC treatment.

Second, our landmark analyses also suggest that response evaluations at delayed time points after TARE should be considered. Indeed, the proportion of patients who achieved CR $(n=3$ [2.9\%] at 1 month; $n=12$ [11.8\%] at 3 months) and PR ( $n=28$ [27.5\%] at 1 month; $n=35$ [34.3\%] at 3 months) by mRECIST increased over time. In agreement with our findings, Vouche et al. ${ }^{33}$ showed that the proportion of patients who achieved CR based on enhancement criteria increased over time, which indicates the clinical significance of response evaluations at delayed time points after TARE. In cases of external radiation, Kim et al. ${ }^{34}$ showed that response evaluations based on enhancement criteria showed better predictions of pathologic response, and their findings were obtained from surgical specimens after radiotherapy plus concurrent hepatic arterial infusion chemotherapy. In this study, the correlation of the enhancementbased response with the degree of pathological tumor response gradually increased, until 6 months after radiation. All of these findings suggest that the clinical decision to maintain or switch treatment modality can be delayed until 3 to 6 months after the radiation-based treatment was performed for HCC.

Third, in our study, PD was a strong predictor of poor survival by both criteria at all-time points. The augmented arterial enhancement that is observed in the peritumoral area after TARE can sometimes be an important confounding factor. ${ }^{26,27}$ However, if new intrahepatic or extrahepatic arterial enhanced lesions outside of the primary lesion are observed, which indicate local control failure, it is not difficult to confirm PD. The failure of HCC local control with stage migration has been one of the most important predictors of poor survival, ${ }^{35}$ in support of our findings. Recently, Khor et al. ${ }^{36}$ reported that the majority PD patterns after TARE revealed the development of new intrahepatic lesions predominantly in Asian patients with HCC. Similarly, most patients with PD by mRECIST $(n=28,93.3 \%)$ had intrahepatic or extrahepatic progression, rather than primary lesion progression. All of these findings suggest that PD after TARE, especially PD with new lesions outside of the primary tumor lesion, should be regarded as insufficient responses to TARE and a prerequisite for additional or alternative treatments, even at the early time points.

Despite the strengths of our study, several issues remain unresolved. First, due to the retrospective nature of the study, patients might not be representative of real-world situations. Due to the low probability of adverse events after treatment with TARE, a significant proportion of patients preferred TARE than surgical resection or other intra-arterial therapies, and this high-selection might have biased the survival of our study participants: indeed, the survival rates of our study seems longer (mean, 55.5 months) than those of other studies of TARE (about

36 months of mean OS and about 10 to 17 months of median OS) that have been reported in literature. ${ }^{6,710,11}$ Furthermore, other bi-dimensional enhancement criteria were not evaluated: however, in several previous studies, simplified one-dimensional assessments that use mRECIST have been shown to be sufficient prognosticators in patients treated with TACE. ${ }^{22,37}$ Finally, the association between radiological and pathological treatment responses could not be assessed. Further studies that recruit patients who received surgical resection after TARE can resolve this issue.

In conclusion, the response evaluations that used mRECIST were more prognostic than those that used RECIST 1.1 in patients with HCC who were treated with TARE. Clinical decisions to wait for the maximal response to TARE or to add or switch to another therapeutic modality might be delayed until 3 months after TARE, based on mRECIST criteria.

\section{CONFLICTS OF INTEREST}

No potential conflict of interest relevant to this article was reported.

\section{ACKNOWLEDGEMENTS}

This study was supported by the Basic Science Research Program, through the National Research Foundation of Korea, and was funded by the Ministry of Science, ICT, and Future Planning (grant number: 2016R1A1A1A05005138).

\section{AUTHOR CONTRIBUTIONS}

Data acquisition, statistical analysis: J.S.L., H.J.C. Data analysis and interpretation: J.S.L., H.J.C., S.U.K., H.R. Study concept, design, and supervision: S.U.K., H.R. Manuscript drafting: J.S.L., H.J.C., S.U.K., H.R. Critical manuscript revision: S.U.K., H.R., B.K.K., J.Y.P., D.Y.K., S.H.A., K.H.H., Y.E.C., S.E.B., M.S.P., M.J.K. Approval of final manuscript: all authors.

\section{ORCID}

Jae Seung Lee

Hong Jun Choi

Beom Kyung Kim

Jun Yong Park

Do Young Kim

Sang Hoon Ahn

Kwang-Hyub Han

Song-Ee Baek

Yong Eun Chung

Mi-Suk Park

Myeong-Jin Kim

Hyungjin Rhee https://orcid.org/0000-0002-2371-0967

https://orcid.org/0000-0002-3753-4343

https://orcid.org/0000-0002-5363-2496

https://orcid.org/0000-0001-6324-2224

https://orcid.org/0000-0002-8327-3439

https://orcid.org/0000-0002-3629-4624

https://orcid.org/0000-0003-3960-6539

https://orcid.org/0000-0001-8146-2570

https://orcid.org/0000-0003-0811-9578

https://orcid.org/0000-0001-5817-2444

https://orcid.org/0000-0001-7949-5402

https://orcid.org/0000-0001-7759-4458 


\section{REFERENCES}

1. McGlynn KA, Petrick JL, London WT. Global epidemiology of hepatocellular carcinoma: an emphasis on demographic and regional variability. Clin Liver Dis 2015;19:223-238.

2. Korean Liver Cancer Association; National Cancer Center. 2018 Korean Liver Cancer Association-National Cancer Center Korea Practice Guidelines for the management of hepatocellular carcinoma. Gut Liver 2019;13:227-299.

3. Sotiropoulos GC, Lang H, Frilling A, et al. Resectability of hepatocellular carcinoma: evaluation of 333 consecutive cases at a single hepatobiliary specialty center and systematic review of the literature. Hepatogastroenterology 2006;53:322-329.

4. Kim JY, Sinn DH, Gwak GY, et al. Transarterial chemoembolization versus resection for intermediate-stage (BCLC B) hepatocellular carcinoma. Clin Mol Hepatol 2016;22:250-258.

5. Sangro B, Salem R. Transarterial chemoembolization and radioembolization. Semin Liver Dis 2014;34:435-443.

6. Salem R, Lewandowski RJ, Mulcahy MF, et al. Radioembolization for hepatocellular carcinoma using yttrium-90 microspheres: a comprehensive report of long-term outcomes. Gastroenterology 2010;138:52-64.

7. Hilgard P, Hamami M, Fouly AE, et al. Radioembolization with yttrium-90 glass microspheres in hepatocellular carcinoma: European experience on safety and long-term survival. Hepatology 2010;52:1741-1749.

8. Kulik LM, Carr BI, Mulcahy MF, et al. Safety and efficacy of $90 \mathrm{Y}$ radiotherapy for hepatocellular carcinoma with and without portal vein thrombosis. Hepatology 2008;47:71-81.

9. Kim HC. Radioembolization for the treatment of hepatocellular carcinoma. Clin Mol Hepatol 2017;23:109-114.

10. Mazzaferro V, Sposito C, Bhoori S, et al. Yttrium-90 radioembolization for intermediate-advanced hepatocellular carcinoma: a phase 2 study. Hepatology 2013;57:1826-1837.

11. Sangro B, Carpanese L, Cianni R, et al. Survival after yttrium-90 resin microsphere radioembolization of hepatocellular carcinoma across Barcelona clinic liver cancer stages: a European evaluation. Hepatology 2011;54:868-878.

12. Gabr A, Abouchaleh N, Ali R, et al. Outcomes of surgical resection after radioembolization for hepatocellular carcinoma. J Vasc Interv Radiol 2018;29:1502-1510.

13. Song JE, Jung KS, Kim DY, et al. Transarterial radioembolization versus concurrent chemoradiation therapy for locally advanced hepatocellular carcinoma: a propensity score matching analysis. Int J Radiat Oncol Biol Phys 2017;99:396-406.

14. Miller AB, Hoogstraten B, Staquet M, Winkler A. Reporting results of cancer treatment. Cancer 1981;47:207-214.

15. Therasse P, Arbuck SG, Eisenhauer EA, et al. New guidelines to evaluate the response to treatment in solid tumors. European Organization for Research and Treatment of Cancer, National Cancer
Institute of the United States, National Cancer Institute of Canada. J Natl Cancer Inst 2000;92:205-216.

16. Lencioni R, Llovet JM. Modified RECIST (mRECIST) assessment for hepatocellular carcinoma. Semin Liver Dis 2010;30:52-60.

17. Kim SU, Kim BK, Han KH. Clinical application of liver stiffness measurement using transient elastography: a surgical perspective. Digestion 2013;88:258-265.

18. Kim MN, Kim BK, Han KH, Kim SU. Evolution from WHO to EASL and mRECIST for hepatocellular carcinoma: considerations for tumor response assessment. Expert Rev Gastroenterol Hepatol 2015;9:335-348.

19. Llovet JM, Di Bisceglie AM, Bruix J, et al. Design and endpoints of clinical trials in hepatocellular carcinoma. J Natl Cancer Inst 2008;100:698-711.

20. Jeon MY, Lee HW, Kim BK, et al. Reproducibility of European Association for the Study of the Liver criteria and modified Response Evaluation Criteria in Solid Tumors in patients treated with sorafenib. Liver Int 2018;38:1655-1663.

21. Kim BK, Kim SU, Kim MJ, et al. Number of target lesions for EASL and modified RECIST to predict survivals in hepatocellular carcinoma treated with chemoembolization. Clin Cancer Res 2013;19:1503-1511.

22. Kim BK, Kim KA, Park JY, et al. Prospective comparison of prognostic values of modified Response Evaluation Criteria in Solid Tumours with European Association for the Study of the Liver criteria in hepatocellular carcinoma following chemoembolisation. Eur J Cancer 2013;49:826-834.

23. Kim BK, Kim SU, Kim KA, et al. Complete response at first chemoembolization is still the most robust predictor for favorable outcome in hepatocellular carcinoma. J Hepatol 2015;62:1304-1310.

24. Weng Z, Ertle J, Zheng S, et al. Choi criteria are superior in evaluating tumor response in patients treated with transarterial radioembolization for hepatocellular carcinoma. Oncol Lett 2013;6:1707-1712.

25. Riaz A, Gabr A, Abouchaleh N, et al. Radioembolization for hepatocellular carcinoma: statistical confirmation of improved survival in responders by landmark analyses. Hepatology 2018;67:873883.

26. Bester L, Hobbins PG, Wang SC, Salem R. Imaging characteristics following 90yttrium microsphere treatment for unresectable liver cancer. J Med Imaging Radiat Oncol 2011;55:111-118.

27. Semaan S, Makkar J, Lewis S, Chatterji M, Kim E, Taouli B. Imaging of hepatocellular carcinoma response after (90)y radioembolization. AJR Am J Roentgenol 2017;209:W263-W276.

28. Kim TH, Kim SY, Tang A, Lee JM. Comparison of international guidelines for noninvasive diagnosis of hepatocellular carcinoma: 2018 update. Clin Mol Hepatol 2019;25:245-263.

29. Korean Liver Cancer Study Group (KLCSG); National Cancer Center, Korea (NCC). 2014 KLCSG-NCC Korea practice guideline for the management of hepatocellular carcinoma. Gut Liver 2015;9:267-317.

30. Mitchell DG, Bruix J, Sherman M, Sirlin CB. LI-RADS (Liver Imag- 
ing Reporting and Data System): summary, discussion, and consensus of the LI-RADS Management Working Group and future directions. Hepatology 2015;61:1056-1065.

31. Lewandowski RJ, Sato KT, Atassi B, et al. Radioembolization with 90Y microspheres: angiographic and technical considerations. Cardiovasc Intervent Radiol 2007;30:571-592.

32. Eisenhauer EA, Therasse P, Bogaerts J, et al. New response evaluation criteria in solid tumours: revised RECIST guideline (version 1.1). Eur J Cancer 2009;45:228-247.

33. Vouche M, Kulik L, Atassi R, et al. Radiological-pathological analysis of WHO, RECIST, EASL, mRECIST and DWI: imaging analysis from a prospective randomized trial of Y90 \pm sorafenib. Hepatology 2013;58:1655-1666.

34. Kim JW, Seong J, Park MS, et al. Radiological-pathological correlation study of hepatocellular carcinoma undergoing local chemo- radiotherapy and surgery. J Gastroenterol Hepatol 2016;31:16191627.

35. Bibault JE, Dewas S, Vautravers-Dewas C, et al. Stereotactic body radiation therapy for hepatocellular carcinoma: prognostic factors of local control, overall survival, and toxicity. PLoS One 2013;8:e77472.

36. Khor AY, Toh Y, Allen JC, et al. Survival and pattern of tumor progression with yttrium-90 microsphere radioembolization in predominantly hepatitis B Asian patients with hepatocellular carcinoma. Hepatol Int 2014;8:395-404.

37. Sato $\mathrm{Y}$, Watanabe $\mathrm{H}$, Sone $\mathrm{M}$, et al. Tumor response evaluation criteria for HCC (hepatocellular carcinoma) treated using TACE (transcatheter arterial chemoembolization): RECIST (Response Evaluation Criteria in Solid Tumors) version 1.1 and mRECIST (modified RECIST): JIVROSG-0602. Ups J Med Sci 2013;118:16-22. 